\title{
Growth, Quality Characterization and Mechanical Hardness of DAST Crystals
}

\author{
E. Dolzhenkova ${ }^{a *}$, G. Babenko ${ }^{a}$, A. Voronov ${ }^{a}$, I. Pritula $^{a}$, \\ A.G. Fedorov ${ }^{a}$, R. GAlbadrakH ${ }^{b}$ And L. ENKHTOR ${ }^{b}$ \\ ${ }^{a}$ Institute for Single Crystals NASU, 60 Nauki ave., 61001 Kharkov, Ukraine \\ ${ }^{b}$ Department of Physics, School of Science, National University of Mongolia, \\ 1 Ikh Surguuliin gudamj, Baga toiruu, Sukhbaatar district, Ulaanbaatar, Mongolia
}

Received: 04.06.2021 \& Accepted: 13.12.2021

Doi: $10.12693 /$ APhysPolA.141.41

*e-mail: complex.borate@gmail.com

\begin{abstract}
4- $N, N^{\prime}$-dimethylamino- $N$-methyl- $4^{\prime}-N^{\prime}$-stilbazolium tosylate crystals were grown by spontaneous crystallization and onto a seed using controlled slow cooling technique. The surface morphology of the grown crystals has been studied. As shown by X-ray diffraction analysis, the perfection of the structure of the grown plates depends on the method of obtaining them. The grown crystals were also characterized by the Vickers microhardness test and etching investigations. The values of microhardness for the crystals grown by spontaneous crystallization are found to exceed the corresponding values for the crystals grown onto a seed by $9-11 \%$. There was established a correlation between the values of microhardness and the character of dislocations distribution in the crystals obtained by both methods. An assumption was made about the $4-N, N^{\prime}$-dimethylamino- $N$-methyl- $4^{\prime}-N^{\prime}$-stilbazolium tosylate deformation mechanism.
\end{abstract}

topics: organic compounds, growth from solutions, characterization, etching

\section{Introduction}

Effective and dynamically developing fields of physics such as quantum electronics and photonics call for creation of new materials characterized by high nonlinear optical coefficients [1]. Inorganic $\mathrm{LiNbO}_{3}, \mathrm{KTP}$, and KDP crystals for a long time used in nonlinear optics and optoelectronics have already reached their efficiency threshold $[2,3]$. During recent years, molecular nonlinear optical crystals arouse a great interest as promising materials for optical communication systems. Due to a small length of chromophores in molecular crystal packing, these crystals can be used at frequencies up to terahertz range. High nonlinear optical susceptibility of the molecular structures is due to the effect of strong polarizability of a cloud of $\pi$-electrons in the conjugate system of organic molecules. The electrooptical coefficient of the molecular crystals is in the range of $100-70 \mathrm{pm} / \mathrm{V}$, which by an order exceeds the corresponding parameters of known inorganic materials [4].

4 - $N, N^{\prime}$-dimethylamino- $N$-methyl- $4^{\prime}-N^{\prime}$-stilbazolium tosylate (DAST) crystals possess unique properties in the optical range (the coefficient of nonlinearity $d_{11}=1010 \pm 110 \mathrm{pm} / \mathrm{V}$ at $\lambda=1318 \mathrm{~nm}$ ) [5]. These crystals show the maximal electrooptical sensitivity $\left(r_{11}=400 \pm 150 \mathrm{pm} / \mathrm{V}\right.$ at $\lambda=820 \mathrm{~nm} ; r_{11}=47 \pm 8 \mathrm{pm} / \mathrm{V}$ at $\lambda=1535 \mathrm{~nm}$ ) and looks promising for optoelectronic processing of terahertz range signals [6]. In the technologically significant range from $0.75 \mu \mathrm{m}$ to $1.65 \mu \mathrm{m}$, the DAST crystals are characterized by a good spectral transmission [7]. Due to their high thermal stability (at temperatures up to $300^{\circ} \mathrm{C}$ ) these crystals can be used in the data transmission systems [8].

DAST belongs to the monoclinic space group $C_{c}$ (point group $m, Z=4$ ) with the lattice parameters $a=10.365 \AA, b=11.322 \AA, c=17.893 \AA$, and $\beta=92.24^{\circ}$ [9]. The molecular packing of DAST crystals is defined by stilbazolium, the cation, one of the most efficient chromophores, and tosylate, the anion, which form a non-centrosymmetric crystal structure.

DAST single crystals are usually grown from a supersaturated solution by the method of slow cooling [9-11]. Several growth methods such as slope nucleation (SNM)-slow cooling (SC) [12, 13], SNMslow evaporation (SE) [8, 14], SNM-seed-crystal method (SCM) [15], seed crystal fixing method (SCF) [16], two zone growth technique (TZGT) [17] have been applied for growing the DAST crystals. The use of this promising material is limited by the difficulties in obtaining homogeneous defect-free crystals, since such crystals show a noticeable tendency to twinning. 
As a rule, organic crystals belong to soft materials that may complicate the process of optical elements fabrication from the crystals [8, 12-14]. Therefore, the investigation of the mechanical properties of DAST crystals evokes an essential interest. It should be noted that the available literature data on the mechanical properties of these crystals are sparse and often contradictory.

In this paper, we report and analyse the quality characterization study and the Vickers microhardness investigations of the DAST molecular crystals obtained under different conditions.

\section{Experimental}

Growth from low temperature solutions has been found to be the most suitable for obtaining of high quality DAST crystals [9]. The studies of DAST solubility in pure methanol, ethanol, acetonitrile and various mixed solvents were carried out in [13, 18]. As seen from these studies, in ethanol, acetonitrile and in a mixed solvent of methanol-ethanol, ethanol-acetonitrile, DAST showed a rather low solubility. Among pure organic solvents, the highest solubility of DAST was found in methanol. The solubility in the mixed solution of methanol-acetone was on the level of pure methanol [13]. In the binary system methanol-acetonitrile, the solubility increased approximately twice or more than the one in pure methanol [18]. In the case of the methanol solution, the method of slow cooling was used to grow DAST single crystals of a plate-like shape, in which the crystallographic $a$ - and $b$-axes were on the developed plane (001) [13]. The rate of the growth along the $\boldsymbol{a}$ - and the $b$-axes was higher than the one along the $c$-axis. The crystals obtained from the methanol-acetonitrile mixed solution were thicker than those grown from methanol [18]. The rate of the growth along the $a$ - and $b$-axes was equal to that of the growth along the $c$-axis. These results testify the possibility of morphological control of DAST crystals by varying solvents during the growth. The plate-shaped crystals are preferable for efficient terahertz wave generation. As shown in [19], DAST crystals with a thickness of $1.0-1.5 \mathrm{~mm}$ are efficient for the practical $\mathrm{THz}$ application. Methanol was found to be the most suitable solvent for the growth of high-quality DAST crystals applied in electrooptics [9]. DAST was synthesized at Division of Functional Materials Chemistry SSI "Institute for Single Crystals" NAS of Ukraine. The DAST powder crystals used for crystal growth was prepared similar to the described procedure [18] by reaction of 4-methylpyridine, methyl- $p$-toluenesulfonate and 4-dimethylaminobenzaldehyde in dimethylformamide/xylene mixture in the presence of piperidine as a catalyst. Solution containing $3.1 \mathrm{~g}$ $\mathrm{DAST} / 100 \mathrm{~g}$ methanol was heated up to $55^{\circ} \mathrm{C}$ and held at this temperature during $\approx 10 \mathrm{~h}$ at continuous stirring to provide complete dissolution of the substance and ensure its homogeneity. Afterwards the temperature in the crystallizer was lowered to $39^{\circ} \mathrm{C}$ (the equilibrium temperature) at a rate of $0.3^{\circ} \mathrm{C} / \mathrm{h}$ without stirring, and then the solution was held during $6 \mathrm{~h}$ for stabilization. Further, the temperature lowering rate was reduced to $0.5^{\circ} \mathrm{C} /$ day until the DAST crystals were spontaneously nucleated. Afterwards the cooling rate was maintained on a level of $0.2^{\circ} \mathrm{C} /$ day till the end of the crystal growth process.

DAST crystals were also grown onto a seed from supersaturated methanol solution by controlled slow cooling technique in the range from 43 to $30^{\circ} \mathrm{C}$. Seed crystals measuring $\approx 2 \times 2 \mathrm{~mm}$ with a well-defined shape and good transparency were obtained by spontaneous nucleation. The seeds were mounted in a holder and immersed to a solution, the temperature of which was by $1^{\circ}$ higher than the equilibrium temperature. The solution was then slowly cooled to the equilibrium temperature $\left(39^{\circ} \mathrm{C}\right)$ without stirring. During 2 days, the temperature was decreased at a rate of $1^{\circ} \mathrm{C} /$ day and then at a constant rate of $0.1-0.2^{\circ} \mathrm{C} /$ day till the end of the crystallization process. Thereat, the solution was mixed at a rate of $20 \mathrm{rpm}$ with $20 \mathrm{~s}$ reverse. Before changing the direction of rotation, the engine stopped for $7 \mathrm{~s}$. The crystallization period was 30 days.

The perfection of the structure of the grown crystals was identified with X-ray rocking curves ( $\mathrm{RC}$ ) by using the method of two-crystal X-ray diffractometry. The RCs were obtained with a double crystal spectrometer at $\mathrm{Cu} K_{\alpha_{1}}$ radiation with a silicon monochromator adjusted to the (400) reflection. The used arrangement allows to pick out a wellresolved $K_{\alpha_{1}}$ line of the X-ray spectrum. To obtain the RCs in $(n,-m)$ scheme, the $(00 \overline{4})$ reflection of the DAST samples was chosen, due to its Bragg reflection angle nearest to that of (400) Si. This significantly reduced the RC broadening caused by dispersion. The measured values of full width at half maximum (FWHM) can be compared with the calculated ones obtained in accordance with [20].

The structure perfection of the grown crystals was also characterized using the metallographic method. The quality of the crystals was evaluated taking into account the imperfections and dislocations present in the crystal which appeared during the growth period. Dislocations were revealed by selective chemical etching in methanol on the facet parallel to the crystallographic plane (001). After etching, the surface was dried using filter paper. The etching time was $10 \mathrm{~s}$. The revealed dislocation structure was examined by means of a microscope Zeiss Axioskop 40 A POL.

Mechanical characteristics of the DAST crystals were investigated using the Vickers microhardness measurement. Indentation was realized using PMT3 device with a standard tetrahedral indenter, the loads ranged from 1 to $20 \mathrm{~g}$ for indentation time of $10 \mathrm{~s}$. The hardness study was realized on the faces (001) of the grown crystals, the diagonals of 

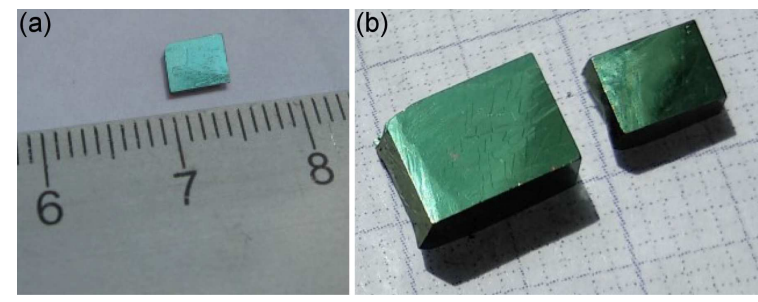

Fig. 1. Photographs of DAST crystals obtained by (a) spontaneous crystallization and (b) grown onto a seed.
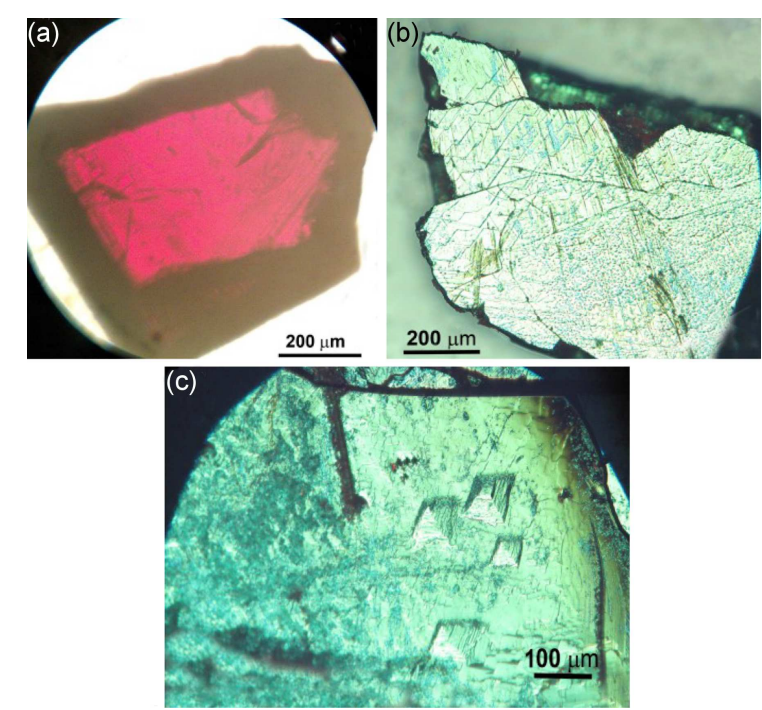

Fig. 2. DAST crystals grown by spontaneous crystallization: transmitted polarized light (a), reflected light: surface (b), growth hillocks (c).

the square impression were parallel to the direction [100]. Prior to the measurements, the investigated surface was polished gently with methanol. The value of microhardness $H_{v}$ was computed using the standard relation [21].

The measurements were realized on 10 imprints at each designated indentation load. The standard deviation of the measured value of mean microhardness was about $2 \%$.

\section{Results and discussion}

Figure 1 presents photographs of DAST crystals obtained from supersaturated methanol solution using slow cooling method by spontaneous crystallization and grown onto a seed. The crystals have the form of plates with a larger surface plane parallel to (001). Their maximum dimensions are $4.5 \times 4 \times 1 \mathrm{~mm}^{3}$ and $8 \times 6 \times 3 \mathrm{~mm}^{3}$, respectively.

The photographs of the DAST crystals grown by the spontaneous crystallization, obtained in the transmitted polarized and in reflected light, are shown in Fig. 2. Examination in the polarized light

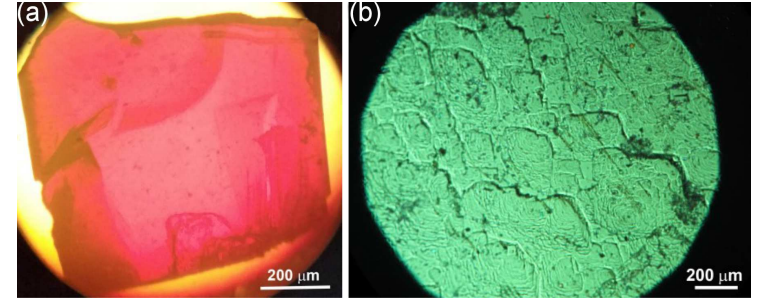

Fig. 3. DAST crystals grown onto a seed using the slow cooling technique: (a) transmitted polarized light, (b) reflected light.

showed that the crystals were optically homogeneous and did not contain mother liquor inclusions (see Fig. 2a). Step-like patterns observed on the crystal surface point to the directions of interatomic bonds in the crystal (see Fig. 2b). The morphology of the growth steps reflects the symmetry of the (001) face. There are also growth hillocks formed on this face, revealing a two-dimensional layer growth mechanism. All the growth spirals have a monoclinic symmetry (see Fig. 2c).

A study of the DAST crystals grown onto a seed in the polarized light shows that they do not contain optical inhomogeneities (see Fig. 3a). The morphological features of the surface of their face (001) differ from those of the relief of the corresponding face of the DAST crystals obtained by spontaneous crystallization. The surface of the investigated crystals is either smooth or has rounded steps with monoclinic symmetry (see Fig. 3b).

Shown in Fig. 4 are the rocking curves of the grown DAST crystals. As one can see, a slight asymmetry is observed for the diffraction curves recorded for the reflection $(00 \overline{4})$ for the crystals obtained by spontaneous crystallization and onto the seed, while individual microblocks are absent. The FWHM value of the rocking curve for the crystals obtained spontaneously is 151 arcsec (see Fig. 4a). For the crystals grown onto the seed, the diffraction maximum of $(00 \overline{4})$ broadens to 323 arcsec (see Fig. 4b). The authors associated a certain decrease in the structure perfection observed in the studied crystals with the presence of stresses around the contact of the growing crystals with the seed.

The observed differences in (001) surface morphology of the crystals grown onto the seed and by spontaneous crystallization (see Figs. $2 \mathrm{~b}$ and $3 \mathrm{~b}$ ) seems to be due to relaxation of the interfacial stresses induced by the seed crystals. Stress relaxation can occur via multiple kink formation of the steps. As a result, roughness of the spiral steps in these crystals is higher than that in the crystals obtained spontaneously under the same conditions. The mean distance between two adjacent kinks on the surface of the former crystals is smaller than the interkink distance in the latter crystals. Deposition of a structural unit on the step in the crystals grown onto the seed will have high probability, 


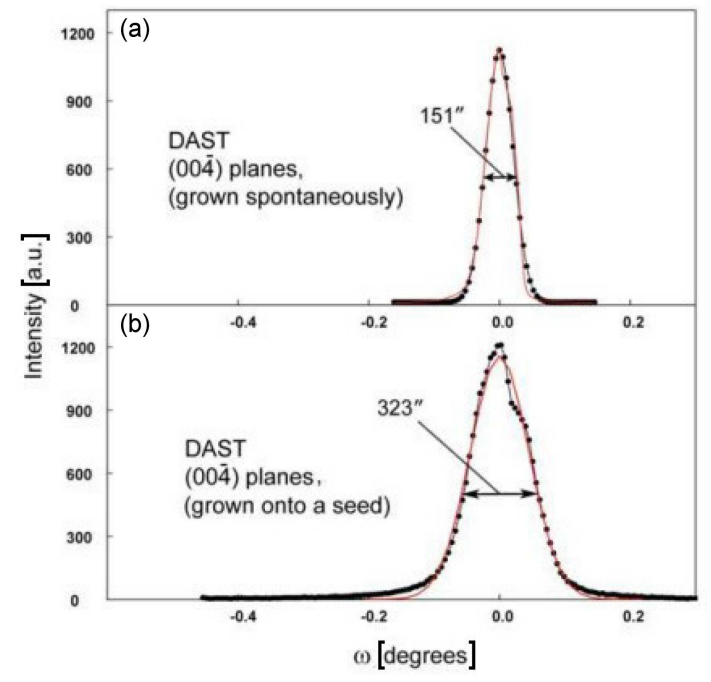

Fig. 4. Rocking curves recorded for DAST single crystals grown by (a) spontaneous crystallization, (b) onto a seed (the thin curves are Gaussian approximation of RCs).

irrespective of the crystallographic direction of the step. Therefore, the step movement is independent of the crystallographic direction and results in the formation of rounded microsteps [22].

Figure 5 presents the photographs of the DAST crystals grown onto a seed at fluctuations of the growth rate. A study of the perfection of such crystals shows that they differ from those obtained without fluctuations of the growth rate. The photographs made in reflected and transmitted polarized light reveal the presence of striations and parasitic crystals on the surfaces of the plate (see Fig. 5a, c). These nonuniformities could arise due to fluctuations of the surface supersaturation [17]. Unlike the samples homogeneous over the whole volume, these crystals have defects in the form of cracks. The latter are propagating in the crystal bulk but do not reach its surface (see Fig. 5b, d). The emergence of such cracks may be due to fluctuations in the growth rate which lead to irregular stacking of the stilbazoliums and the tosylates. Failure in the interleaving of the sheets of both ions along the crystallographic $c$-axis leads to strains in the crystal and to its cracking.

The rocking curve recorded for the crystal containing defects in the form of cracks (see Fig. 6) has five peaks. The full width at a half maximum of the most intensive peaks is 350,178 , and 312 arcsec. The observed additional peaks recorded for $(00 \overline{4})$ diffracting planes depict the presence of an internal structural very low angle grain boundaries in the crystal grown under the conditions of temperature fluctuations. The tilt angles (i.e., misorientation angle between the two crystalline regions on both sides of the structural grain boundary) are $\approx 4$ arcmin.
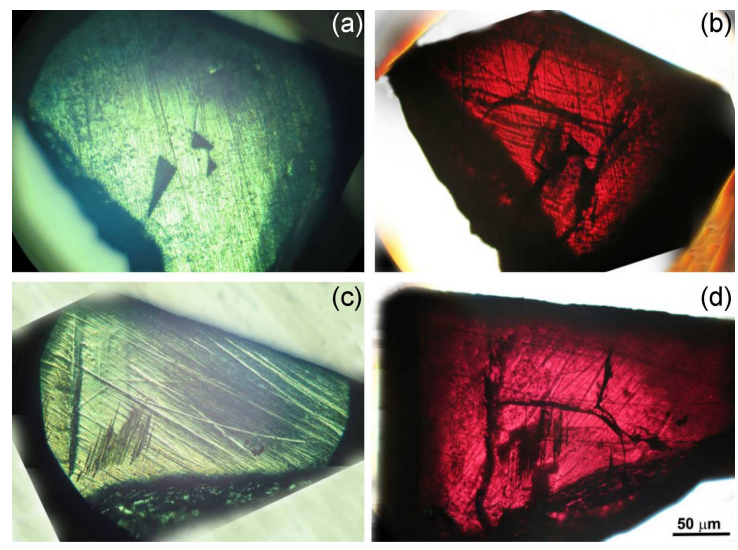

Fig. 5. Defect structure in the bulk of DAST crystal grown onto a seed at fluctuations of the growth rate: $(\mathrm{a}, \mathrm{b})-(001)$ plane, $(\mathrm{c}, \mathrm{d})-(00 \overline{1})$ plane; $(\mathrm{a}, \mathrm{c})$ - reflected light, $(\mathrm{b}, \mathrm{d})$ - transmitted polarized light.

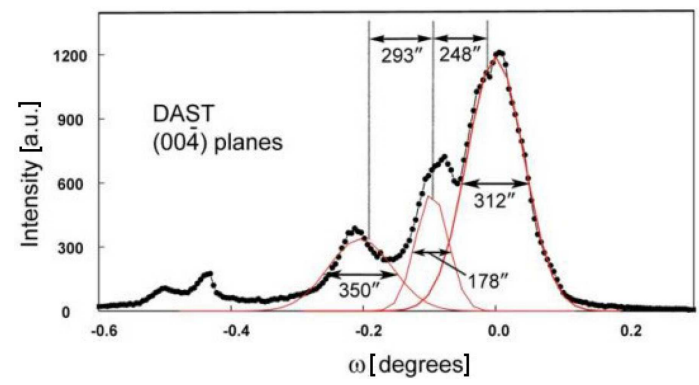

Fig. 6. Rocking curves recorded for DAST single crystals grown onto a seed at fluctuations of the growth rate (the thin curves are Gaussian approximation of RCs).

As found by comparing the half-widths of the rocking curves of the DAST crystals with the corresponding values of other organic crystals [23], FWHM of the former crystals exceeds those of the latter. In particular, the value of FWHM of the rocking curve for LAP crystal is 18.9 arcsec that complies with the corresponding values for inorganic compounds. Such a difference may be explained by the fact that the size of organic molecules in the DAST crystal packing essentially exceeds the size of molecules in LAP packing. The linear dimensions of stilbazolium and tosylate molecules calculated using the program Molecular Orbital PACkage (MOPAC) are $15 \AA \times 6.1 \AA$ and $7.4 \AA \times 5.4 \AA$ (the length and width of stilbazole and tosylate, respectively). For comparison, it should be noted that the linear dimensions of the L-arginine molecules, which together with phosphoric acid and the water molecule form the LAP compound, are $6.3 \AA$ (the length) and $3.1 \AA$ (the thickness) [24].

The crystals containing defects were broken during mechanical treatment as a result of the presence of the cracks in their bulk, and therefore could not be used to make optical elements. Figure 7 


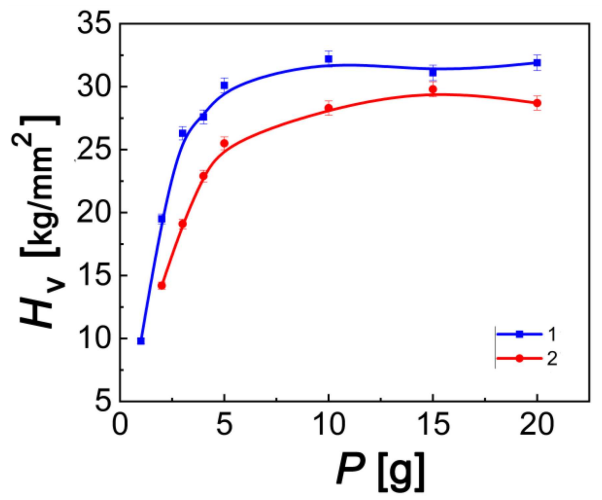

Fig. 7. Loading curve for (001) plane of DAST molecular crystal: 1 - on the surface of a plate grown by spontaneous crystallization, 2 - on the surface of the plate grown onto a seed.

shows the loading curves for the optically homogeneous crystals grown by spontaneous crystallization and onto the seed. The behaviour of the dependence of microhardness on the indenter loading is the same for all the samples: at low loads, the microhardness values rise, whereas at higher loads they remain practically unchanged. The loading curve shows "the reverse indentation size effect". There are two sections on the obtained curves: (i) in 1-5 $\mathrm{g}$ range the value of microhardness essentially depends on the indenter load, (ii) in 10-20 g range such dependence is weak. The microhardness values preserved within the whole interval of the loads seem to be considered an objective characteristic of the resistance of the material to local deformation. Currently, the origin of the "reverse indentation size effect" observed in the DAST crystal has not been analyzed. The Vickers microhardness is calculated using the method of restored imprint. The dimensions of both diagonals $d$ of the imprints are measured after unloading the indenter. Thereat, the elastic component of the deformation under the indenter is not taken into account. Contributions of the elastic and plastic deformations to the total deformation at the low loads are comparable. It may be assumed that the "reverse indentation size effect" is associated with the instrument loading error.

The microhardness average values obtained for the crystals grown spontaneously exceeded the values for the crystals grown onto a seed, and they were respectively equal to 32.6 and $28.1 \mathrm{kgf} / \mathrm{mm}^{2}$ (see Fig. 7, (1), (2)). The comparison was made for the microhardness values which were preserved in the 5-20 g loading interval. Such results testify that the studied DAST crystals belong to soft materials, despite the presence of ionic bonds in the given compound.

Hardness is the ability of materials to resist plastic deformation during indentation. At present, the mechanism of the plastic deformation of the DAST has not been determined, but generally, the process

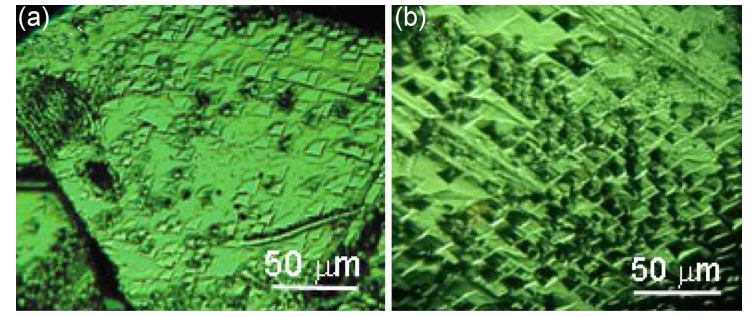

Fig. 8. Etch pits pattern revealed on (001) surface of DAST crystals, obtained by (a) spontaneous crystallization and (b) onto a seed.

of deformation of molecular organic compounds is of dislocation type. From the literature, there are known only few studies devoted to the description of the said defects in dimethylamino stilbazolium tosylate [13]. Therefore, it is a matter of considerable interest to study the dislocation structure and the character of distribution of linear defects in the molecular DAST crystals.

In Fig. 8 there are presented the etch patterns revealed on the face (001) of the grown crystals. The etch pits are either bright with plain bottoms or dark with sharp peaks. Their morphology corresponds to the crystallographic symmetry of the examined plane and has the form of irregular parallelograms. Therefore, it can be assumed that the dark etches pits with peaks corresponding to the emergence of dislocations on the face under consideration. The plain bright pits most probably correspond to the clusters of point defects and reveal the spots of aggregation of the point defects.

The patterns of the etch pits obtained on the surface of the DAST crystal grown by spontaneous crystallization differ from those of the crystals grown onto a seed. On the face (001) of the spontaneously crystallized samples, there were mainly observed the light etch pits and only few dark ones (see Fig. 8a). In turn, on the surface of the crystals grown on the seed, there were numerous dark etch pits and only a few light ones (see Fig. 8b). The dislocation density of the crystals grown onto a seed was equal to $5.7 \times 10^{4} \mathrm{~cm}^{-2}$. The dislocation density of the crystals obtained by spontaneous crystallization was $3.4 \times 10^{2} \mathrm{~cm}^{-2}$. Moreover, the surfaces of such crystals contained a considerable number of point defects.

The values of microhardness of the crystals obtained spontaneously is by $9-11 \%$ higher than those of the crystals grown onto a seed. The mechanical strength is directly related to the mobility of the dislocations in the studied material. Resistance to the dislocation motion in the lattice is due to interactions with other dislocations, point and surface defects. Information concerning the behaviour of dislocations in the DAST crystals is very scarce. It can be assumed that the higher plasticity of the crystal grown onto a seed is due to the fact that the main deformation mechanism is not slipping, which 
is limited in the forest dislocation, but the climbing. The point-defect clusters in the crystals with ionic bonds are effective stoppers for the dislocation motion. This, apparently, explains the observed hardening of the crystal obtained spontaneously.

\section{Conclusion}

DAST crystals with the dimensions $4.5 \times 4 \times$ $1 \mathrm{~mm}^{3}$ and $8 \times 6 \times 3 \mathrm{~mm}^{3}$ were obtained by spontaneous crystallization and grown onto a seed from a supersaturated solution using a controlled slow cooling technique. These crystals had the form of plates parallel to the plane (001). The surface (001) of the crystals has the form of steps with monoclinic symmetry. As shown by X-ray diffraction analysis, the structure perfection of the crystals obtained by the spontaneous crystallization exceeds the corresponding characteristic of the samples grown onto a seed. The average microhardness values of the samples obtained by spontaneous crystallization are by $9-11 \%$ higher than the corresponding values of the crystals grown onto a seed (31.3 and $28.1 \mathrm{kgf} / \mathrm{mm}^{2}$ ), respectively. Plates grown by the spontaneous crystallization are characterized by extremely low dislocation density, namely $3.4 \times 10^{2} \mathrm{~cm}^{-2}$. In the crystals grown onto a seed, the dislocation density is essentially higher and equals $5.7 \times 10^{4} \mathrm{~cm}^{-2}$. The higher plasticity of crystals grown onto a seed might be attributed to the fact that the main mechanism of dislocation movement in DAST, along with slipping, is climbing.

\section{Acknowledgments}

The authors gratefully acknowledge the National University of Mongolia for their financial support through the grant P2019-3741.

\section{References}

[1] T. Kaino, S. Tomaru, T. Kurihara, M. Asobe, Nonlin. Opt. 13, 203 (1996).

[2] H. Hirori, A. Doi, F. Blanchard, K. Tanaka, Appl. Phys. Lett. 98, 091106 (2011).

[3] Yuye Wang, Jianquan Yao, Degang Xu, $\mathrm{Pu}$ Zhao, Peng Wang, Chin. Opt. Lett. 4, 419 (2006).

[4] M.R. Manikandan, S.D. Gopal Ram, G. Ravi, Adv. Mater. Res. 584, 42 (2012).

[5] M. Jazbinsek, L. Mutter, P. Gunter, IEEE J. Sel. Top. Quant. Electron. 14, 1298 (2008).

[6] A. Schneider, I. Biaggio, P. Günter, Opt. Commun. 224, 337 (2003).
[7] S.R. Marder, J.W. Perry, Ch.P. Yakymyshyn, Chem. Mater. 6, 1137 (1994).

[8] R.J. Vijay, N. Melikechi, T. Thomas, R. Gunaseelan, M.A. Arockiaraj, P. Sagayaraj, Mater. Chem. Phys. 132, 610 (2012).

[9] B. Ruiz, M. Jazbinsek, P. Günter, Cryst. Growth Des. 8, 4173 (2008).

[10] S. Brahadeeswaran, S. Onduka, M. Takagi, Cryst. Growth Des. 6, 2463 (2006).

[11] Lifeng Cao, Bing Teng, Ke Feng, Degao Zhong, Lun Hao, Qing Sun, J. Cryst. Growth 412, 20 (2015).

[12] Hui Xu, Bing Teng, Zhenghe Yu, Fei You, De-Gao Zhong, Shi-Ming Zhang, Yue-Juan Yu, Mater. Sci. Forum 687, 447 (2011).

[13] M. Rathnakumari, J. Rajeev Gandhi, L. Mariappan, P. Muralimanohar, K. Sathyamoorthy, P. Suresh Kumar, Int. J. Mater. Sci. 9, 47 (2014).

[14] K. Jagannathan, S. Kalainathan, T. Gnanasekaran, Mater. Lett. 61 , 4485 (2007).

[15] M. Manivannan, S.A. Martin Britto Dhas, M. Jose, J. Inorg. Organomet. Polym. 27 (2017).

[16] S. Sohma, H. Takahashi, T. Taniuchi, H. Ito, Chem. Phys. 245, 359 (1999).

[17] A.S. Haja Hameed, S. Rohani, W.C. Yu, C.Y. Tai, C.W. Lan, J. Cryst. Growth 297, 146 (2006).

[18] Y. Mineno, T. Matsukawa, S. Ikeda et al., Mol. Cryst. Liq. Cryst. 463, 55/[337] (2007).

[19] M. Yoshimura, R. Sakae, Y. Takahashi et al., in: Proc. IEEE 2013 Conf. on Lasers and Electro-Optics Pacific Rim (CLEOPR), 2013, WPC_18.

[20] A.H. Compton, S.K. Allison, X-ray in Theory and Experiment, 2nd ed., van Nostrand, New York 1967.

[21] B.W. Mott, Microindentation Hardness Testing, Butterworth, London 1956.

[22] K. Sangwal, Prog. Cryst. Growth Charact. 19, 189 (1989).

[23] A.S. Haja Hameeda, G. Ravi, R. Ilangovan, A. Nixon Azariah, P. Ramasamy, J. Cryst. Growth 237-239, 890 (2002).

[24] J.J.P. Stewart, MOPAC2016, Stewart Computational Chemistry, Colorado Springs (CO) 2016. 